\title{
On the structure of an aqueous propylene glycol solution
}

\author{
Natasha H. Rhys, ${ }^{1}$ Richard J. Gillams, ${ }^{1}$ Louise E. Collins, ${ }^{2}$ Samantha K. Callear, ${ }^{3}$ M. Jayne Lawrence, ${ }^{2}$, a) and \\ Sylvia E. McLain ${ }^{1, b)}$ \\ ${ }^{1)}$ Department of Biochemistry, University of Oxford, Oxford OX1 $3 Q U U K$ \\ ${ }^{2)}$ Institute of Pharmaceutical Science, King's College London, London SE1 9NH UK \\ ${ }^{3)}$ STFC, ISIS Facility, Rutherford Appleton Laboratory, Didcot OX11 OQU UK
}

(Dated: 25 October 2016)

Using a combination of neutron diffraction and EPSR computational modelling, the interactions in a 30 mol\% aqueous solution of propylene glycol (PG), which govern both the hydration and association of this molecule in solution, have been assessed. From this work it appears that PG is readily hydrated, where the most prevalent hydration interactions were found to be through both the PG hydroxyl groups but also alkyl groups typically considered hydrophobic. Hydration interactions of PG dominate the solution, over PG self-self interactions and there is no evidence of more extensive association. This hydration behavior for PG in solutions suggest that the preference of $\mathrm{PG}$ to be hydrated rather than self associate may translate into a preference for PG to bind to lipids rather than itself, providing a potential explanation for how $\mathrm{PG}$ is able to enhance the apparent solubility of drug molecules in vivo.

PACS numbers: 61.20.-p

\section{INTRODUCTION}

Propylene glycol (1,2-propanediol; PG) is a viscous liquid used in a diverse range of industrial applications including the pharmaceutical, cosmetic and food industries. With a chiral carbon centre, propylene glycol is able to exist in both $\mathrm{R}$ - and $\mathrm{S}$ - enantiomeric forms and has two -OH groups by which it can, in principle, participate in hydrogen bonding interactions. Whilst it is the $\mathrm{R}$ - form commonly produced by micro-organisms and the $\mathrm{S}$ - variant arises naturally from the conversion of sugars, ${ }^{1}$ the commercial product is a racemic mixture of the two, which can be easily synthesised from propylene oxide ${ }^{2}$ or glycerol. ${ }^{3}$

Like similar polyols, propylene glycol is able to depress the freezing point of water, appproaching $-60^{\circ} \mathrm{C}$ at a eutectic composition of 60:40 weight percent ratio of propylene glycol to water. ${ }^{4-6}$ This property allows propylene glycol to be used as a less toxic option for antifreeze solutions, whilst much research is investigating its potential role in cryopreservation of tissues. ${ }^{7-10}$ Recent neutron diffraction studies on cryoprotective solutions at $77 \mathrm{~K}$ suggest that solutions containing propylene glycol as a constituent are arranged such that the water molecules are segregated into percolating clusters, preventing the formation of ice crystals. ${ }^{11}$ How propylene glycol specifically contributes to this microsegregation of water is not clear as methanol - which is thought sequester water into small percolating strings and clusters ${ }^{12,13}$ and dimethyl sulfoxide (DMSO) - which is thought to form tightly bound globular clusters with water, leaving the tetrahedral network of water largely intact, ${ }^{14,15}$ were also present in this cryoprotective solution.

\footnotetext{
a) jayne.lawrence@kcl.ac.uk

b) sylvia.mclain@bioch.ox.ac.uk
}

In the pharmaceutical industry, propylene glycol is used as a solvent to increase the apparent solubility and the permeation of drugs through lipid membranes ${ }^{16-20}$ and as such is used commonly as a co-solvent in oral, intravenous $^{21-23}$ and topical preparations. ${ }^{18,24}$ Interestingly, encapsulating drugs into PG containing liposomes results in a substantial increase in drug-solubility which increases with PG concentration. ${ }^{25}$ The addition of PG to distearoylphosphatidylcholine containing lamellar phases results in an decrease in lamellar thickness and with increasing PG concentrations. ${ }^{21,26}$ Furthermore, computational studies have suggested that $\mathrm{PG}$ induces pore formation in DOPC bilayers at concentrations $\geq 15 \mathrm{~mol} \% .^{27}$

Despite its importance in a range of industrial applications, limited structural information exists for propylene glycol, in comparison to ethylene glycol. ${ }^{28-30}$ The present work explores the structural interactions between propylene glycol and water in solution at a relative molar concentration of 3 propylene glycol : 7 water ( $~ 64 \mathrm{wt} \%$; similar to the eutectic point) using neutron diffraction with isotopic substitution and Empirical Potential Structure Refinement $^{31}$, to produce a model of the system that fits the experimental data. The primary focus of this work is to assess the hydration and association of propylene glycol molecules through both its hydrophilic and hydrophobic groups on the atomic scale in solution.

\section{METHODS}

\section{A. Neutron diffraction}

Neutron diffraction experiments were performed on aqueous propylene glycol solutions (Fig. 2 at a relative molar concentration of 3:7 propylene glycol:water using the SANDALS diffractometer at the ISIS neutron facility (STFC, UK). All experiments were performed at a tem- 


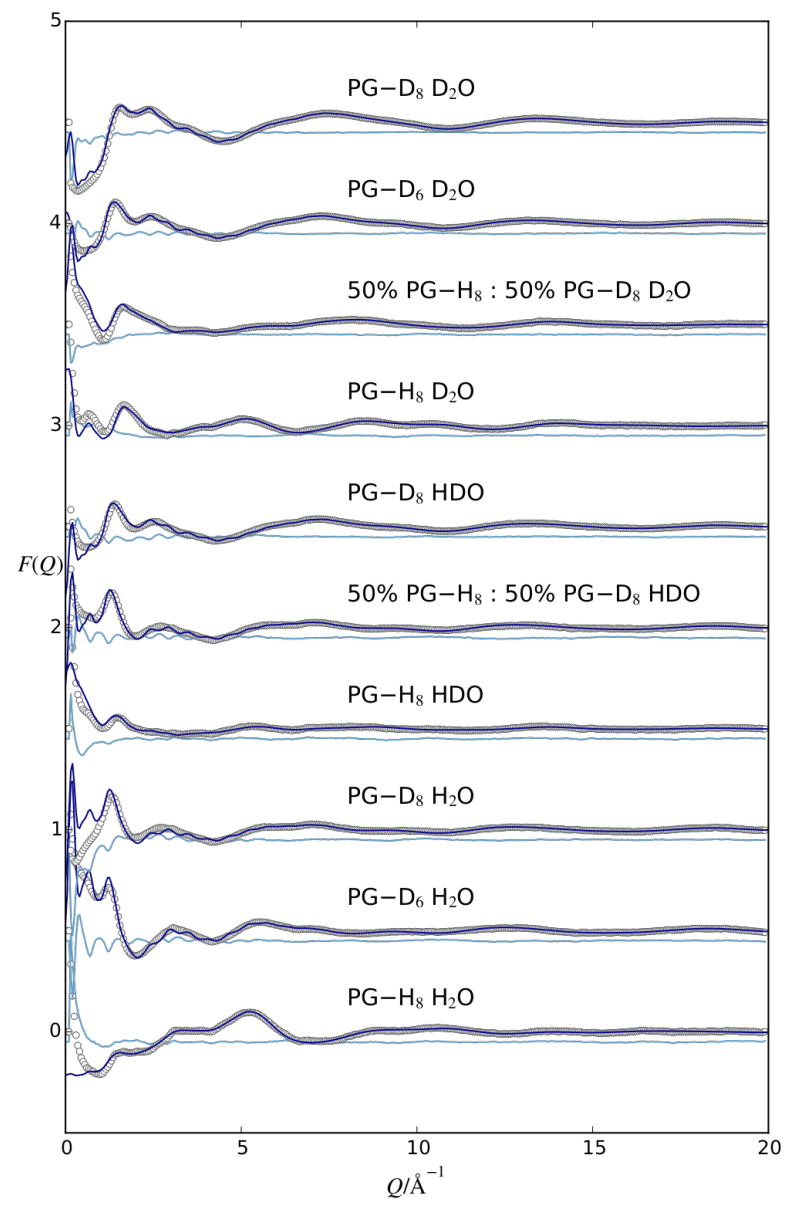

FIG. 1: The $F(Q)$ fits (blue line) to the measured diffraction data (grey circles) and the difference between the fit and the data (grey line) for each of the measured propylene glycol/water solutions. Each dataset has been offset by 0.5 for clarity.

perature of $298 \pm 2 \mathrm{~K}$ and a pressure of 1 bar. Ten isotopomers of the solution were prepared, listed in Table I, using combinations of 1,2-propanediol-h $8\left(\mathrm{PG}-\mathrm{H}_{8}\right), 1,2-$ propanediol- $\mathrm{d}_{6}\left(\mathrm{PG}_{-} \mathrm{D}_{6}\right.$; where only the alkyl hydrogens are deuterated) 1,2-propanediol- $\mathrm{d}_{8}\left(\mathrm{PG}-\mathrm{D}_{8}\right), \mathrm{H}_{2} \mathrm{O}$ and $\mathrm{D}_{2} \mathrm{O} . \mathrm{PG}-\mathrm{H}_{8}, \mathrm{PG}-\mathrm{D}_{6}$ and $\mathrm{D}_{2} \mathrm{O}(99.9 \% \mathrm{D})$ were purchased from Sigma Aldrich and used without further purification and $\mathrm{PG}_{-} \mathrm{D}_{8}$ was purchased from $\mathrm{C} / \mathrm{D} / \mathrm{N}$ isotopes. The propylene glycol isotopomers were all a racemic mixture of R- and S-1,2-propanediol.

The samples were measured in flat-plate cans constructed from a $\mathrm{Ti} / \mathrm{Zr}$ alloy with a sample thickness of $1 \mathrm{~mm}$, to minimize multiple scattering for the protiated samples. Diffraction data for each solution in Table I were collected for an average of $\sim 8$ hours $(\sim 1000 \mu \mathrm{A})$ and were corrected for background, multiple scattering and absorption effects, and normalised using to a vanadium standard to obtain the total static structure factor for each sample, $F(Q)$ using the GUDRUN software available at ISIS. ${ }^{32}$
TABLE I: Isotopomers of $30 \mathrm{~mol} \%$ propylene glycol solutions measured by neutron diffraction

\begin{tabular}{|c|c|c|}
\hline Sample Number & Propylene Glycol & Water \\
\hline $\mathrm{I}$ & $\mathrm{PG}-\mathrm{H}_{8}$ & $\mathrm{H}_{2} \mathrm{O}$ \\
\hline II & PG-D 6 & $\mathrm{H}_{2} \mathrm{O}$ \\
\hline III & PG-D 8 & $\mathrm{H}_{2} \mathrm{O}$ \\
\hline IV & $\mathrm{PG}-\mathrm{H}_{8}$ & $\mathrm{HDO}$ \\
\hline $\mathrm{V}$ & $50 \% \mathrm{PG}^{-\mathrm{H}_{8}}: 50 \% \mathrm{PG}-\mathrm{D}_{8}$ & $\mathrm{HDO}$ \\
\hline VI & $\mathrm{PG}-\mathrm{D}_{8}$ & $\mathrm{HDO}$ \\
\hline VII & $\mathrm{PG}-\mathrm{H}_{8}$ & $\mathrm{D}_{2} \mathrm{O}$ \\
\hline VIII & $50 \% \mathrm{PG}^{-\mathrm{H}_{8}}: 50 \% \mathrm{PG}-\mathrm{D}_{8}$ & $\mathrm{D}_{2} \mathrm{O}$ \\
\hline IX & $\mathrm{PG}-\mathrm{D}_{6}$ & $\mathrm{D}_{2} \mathrm{O}$ \\
\hline $\mathrm{X}$ & PG-D 8 & $\mathrm{D}_{2} \mathrm{O}$ \\
\hline
\end{tabular}

$F(Q)$, which is the sum of all of the pair correlations in the system, can be written as:

$$
F(Q)=\sum_{\alpha, \beta \geq \alpha}\left(2-\delta_{\alpha \beta}\right) c_{\alpha} c_{\beta} b_{\alpha} b_{\beta}\left(S_{\alpha \beta}(Q)-1\right)
$$

where $c$ is concentration of $\alpha$ and $\beta, b$ the coherent neutron scattering length of each, ${ }^{33}$ and $Q$ is the difference between the incident and scattered vector of the neutron probe. $F(Q)$ is the sum of all of the partial correlation functions, $S(Q)$ :

$$
S_{\alpha \beta}(Q)=1+\frac{4 \pi \rho}{Q} \int r\left[\left(g_{\alpha \beta}(r)-1\right)\right] \sin (Q r) \mathrm{d} r
$$

From $S(Q)$, the individual radial distribution function, $g_{\alpha \beta}(r)$ can be obtained via Fourier transformation and integration of this function within distances $r_{1}$ and $r_{2}$ provides the relevant coordination number for the pairwise interactions:

$$
n_{\alpha}^{\beta}=4 \pi \rho c_{\beta} \int_{r_{1}}^{r_{2}} r^{2} g_{\alpha \beta}(r) \mathrm{d} r
$$

$F(Q)$ for each measured isotopomer listed in Table I are shown in Fig. 1 and the corresponding Fourier transformation of this total scattering data $G(r)$ which is the summation of all of the $g(r)$ s weighted by concentration and scattering length are shown in the Supplementary Information.

\section{B. Empirical Potential Structure Refinement}

In order to extract a full set of correlations between propylene glycol and water, Empirical Potential Structure Refinement (EPSR) simulations ${ }^{34}$ were performed on a box containing 750 propylene glycol molecules, 375 R-isomers and 375 S-isomers, and 1750 water molecules and the molecular structure and labelling of these molecules are shown in Fig. 2. The central carbon and hydroxyl groups have been labelled differently for each enantiomer. There are fifteen unique 


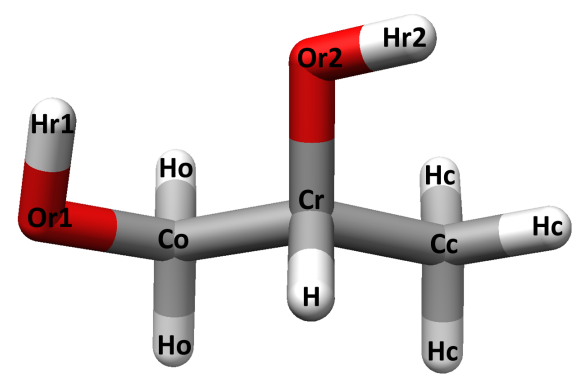

(a)

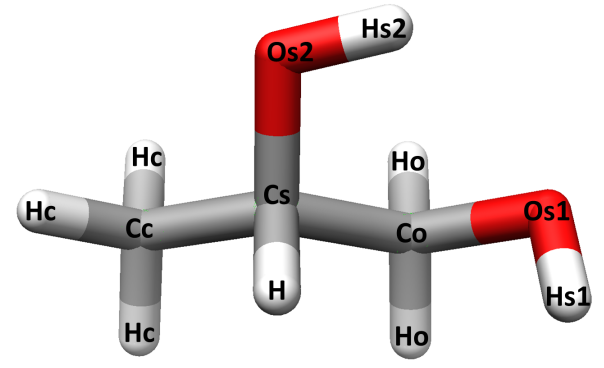

(b)

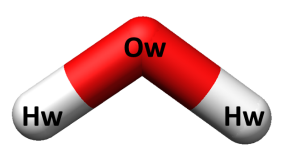

(c)

FIG. 2: Molecular structures of (a) R- and (b) Spropylene glycol and (c) water with the atomic labels used in the EPSR simulation.

atomic site for the propylene glycol molecules, combining labels from both enantiomers, and two for water which gives $153 g(r) \mathrm{s}$, which are impossible to extract from the data alone and as such computational modelling allows for the extraction of the individual site-site correlations.

EPSR has been specifically designed in order to produce a computational model constrained to fit the experimental neutron diffraction data. ${ }^{34,35}$ The use of EPSR to analyse neutron diffraction data has been frequently exploited to study the solution properties of a range of organic liquids, ${ }^{11,12,14,36-46}$ biological and drug molecules $^{47-62}$ and ionic species. ${ }^{63-66} \mathrm{~A}$ set of reference potentials are used to start the EPSR process and these potentials are subsequently modified to provide a fit to the neutron diffraction data through a reverse Monte Carlo process. The Lennard-Jones potentials and Coulombic charges for each atomic component are listed in Table II for both R- and S-propylene glycol molecules, which were obtained from the CHARMM forcefield ${ }^{67}$ and their intramolecular geometry optimised using the Avogadro software ${ }^{68}$ and the SPC/E model was used for the water molecules. ${ }^{69}$ Within the simulation while the angles and bond information was used to define the PG
TABLE II: Lennard-Jones potentials and Coulombic charges for propylene glycol and water used as the reference potential in the EPSR simulation.

\begin{tabular}{cccc}
\hline \hline Atom & $\epsilon\left(\mathrm{kJ} \mathrm{mol}^{-1}\right)$ & $\sigma(\AA)$ & $q_{e}$ \\
\hline$C r$ & 0.13389 & 3.56360 & 0.140 \\
$C s$ & 0.13389 & 3.56360 & 0.140 \\
$C c$ & 0.32635 & 3.65269 & -0.273 \\
$C o$ & 0.23430 & 3.58141 & -0.053 \\
Or 1 & 0.80375 & 3.14487 & -0.649 \\
Or 2 & 0.80375 & 3.14487 & -0.650 \\
Os 1 & 0.80375 & 3.14487 & -0.649 \\
Os2 & 0.80375 & 3.14487 & -0.650 \\
$H$ & 0.18828 & 2.38761 & 0.090 \\
$H c$ & 0.10042 & 2.38761 & 0.090 \\
$H o$ & 0.14644 & 2.38761 & 0.090 \\
$H r 1$ & 0.19246 & 0.40001 & 0.420 \\
$H r 2$ & 0.19246 & 0.40001 & 0.419 \\
$H s 1$ & 0.19246 & 0.40001 & 0.420 \\
$H s 2$ & 0.19246 & 0.40001 & 0.419 \\
\hline$O w$ & 0.65000 & 3.1660 & -0.8476 \\
$H w$ & 0 & 0 & 0.4238 \\
\hline \hline
\end{tabular}

molecules, there were no additional constraints imposed upon the molecular structure of PG - such as dihedrals or rotational groups. It should be noted, however, that in EPSR molecules are not held rigid as they are in many MD simulations but are rather have highly flexible bonds in order to satsifactorily reproduce the experimental neutron diffraction data. ${ }^{70}$ However, the molecular conformation of the PG molecules showed that both enatiomers remained largely in the 'gauche' conformation, which is the energetically most stable configuration of PG molecules (see Supporting information). It should be noted that EPSR does not provide the only possible model for the liquid in question however it does provide a model which is consistent with a set of measured data - where in this case there are 10 unique data sets - and is physically reasonable. It should be noted that the final model is somewhat dependent on the seed potential values, especially in the case where some of the correlations are not well represented in any measured data set. It is for this reason that a wide range of isotopomers are measured, to ensure the most reliable model possible within the constraints of the measurement.

\section{ANGULA}

Supplementary to the $g(r)$ s, three-dimensional arrangements of the molecules around propylene glycol were extracted from the EPSR simulation box using the program ANGULA. ${ }^{48,71}$ For the ANGULA analyses, orthonormal coordinates have been assigned to the water and specific sites on the propylene glycol molecules, by determining a representative centre of mass for the coordinate system. For propylene glycol, these coordinate systems were centered on the oxygen atoms (O1 and O2) 
to characterise hydroxyl interactions and on the methyl carbon (Cc) for alkyl interactions (see Section III of the Supplementary Information). From this analysis, the distribution of the neighboring water molecules or other propylene glycol molecules can be plotted relative to specific sites on a central propylene glycol. By accumulating $\sim 4000$ different snapshots of the simulation box, the nearest neighbor contacts made with functional groups on propylene glycol have here been plotted as a Spatial Density Map (SDM), where the density represent the positions where molecules can be found around a given group. ${ }^{48,72}$ The scale bar for each SDM represents the density of nearest neighbors around a central propylene glycol for all the SDMs presented, which has been normalised according to the number of R- or S- propylene glycol molecules in the simulation box.

\section{RESULTS AND DISCUSSION}

\section{A. Water-water interactions}

Fig. 3 shows the $g(r)$ s for interactions between water molecules in aqueous propylene glycol and Table III gives the coordination numbers for these functions at the minima after the first peak. These functions have been compared to EPSR simulations on pure water. ${ }^{73}$ For each $g(r)$ in Fig. 3 there is an increase in the peak height for all coordination shells. This can be, in part, attributed to excluded volume effects, where the local density around a given water molecule in a short distance range is different than the average bulk density of the solution. ${ }^{14,74}$

Compared to pure water, there is a decrease in the number of hydrogen bonding interactions. For the $\mathrm{Ow}-$ $\mathrm{Hw}$ interaction, the coordination number has decreased by $\sim 40 \%$ to 1.1 , similar to what occurs in concentrated solutions of sorbitol ${ }^{38}$, acetone and DMSO, ${ }^{14}$. While the peak positions of the first coordination shell are similar to those for pure water in Fig. 3, the second coordination shell for the $\mathrm{Ow}-\mathrm{Ow}$ function occurs at a shorter distance of $4.25 \AA$, compared to $4.56 \AA$ in pure water. ${ }^{43,73} \mathrm{~A}$ slight shift in this peak is also observed in the water structure for a number of molecules in aqueous solution, such as methanol ${ }^{12,13}$, glycerol ${ }^{43}$, tertiary butanol, ${ }^{37} \mathrm{DMSO}$, acetone $^{14}$ and the amino acid proline. ${ }^{48,53}$ However, shifts in this peak position are not necessarily indicative of a change in the tetrahedral structure of water, ${ }^{14,75}$ and in the present case there appears to be only a slight perturbation of the bulk water structure, which is likely due to both density effects and possibly slight preference for self-interactions between water molecules.

\section{B. Hydrophilic hydration}

With two hydroxyl groups, propylene glycol can form hydrogen bonds with water molecules both as a donor and as an acceptor.The $g(r) \mathrm{s}$ for the interactions of both
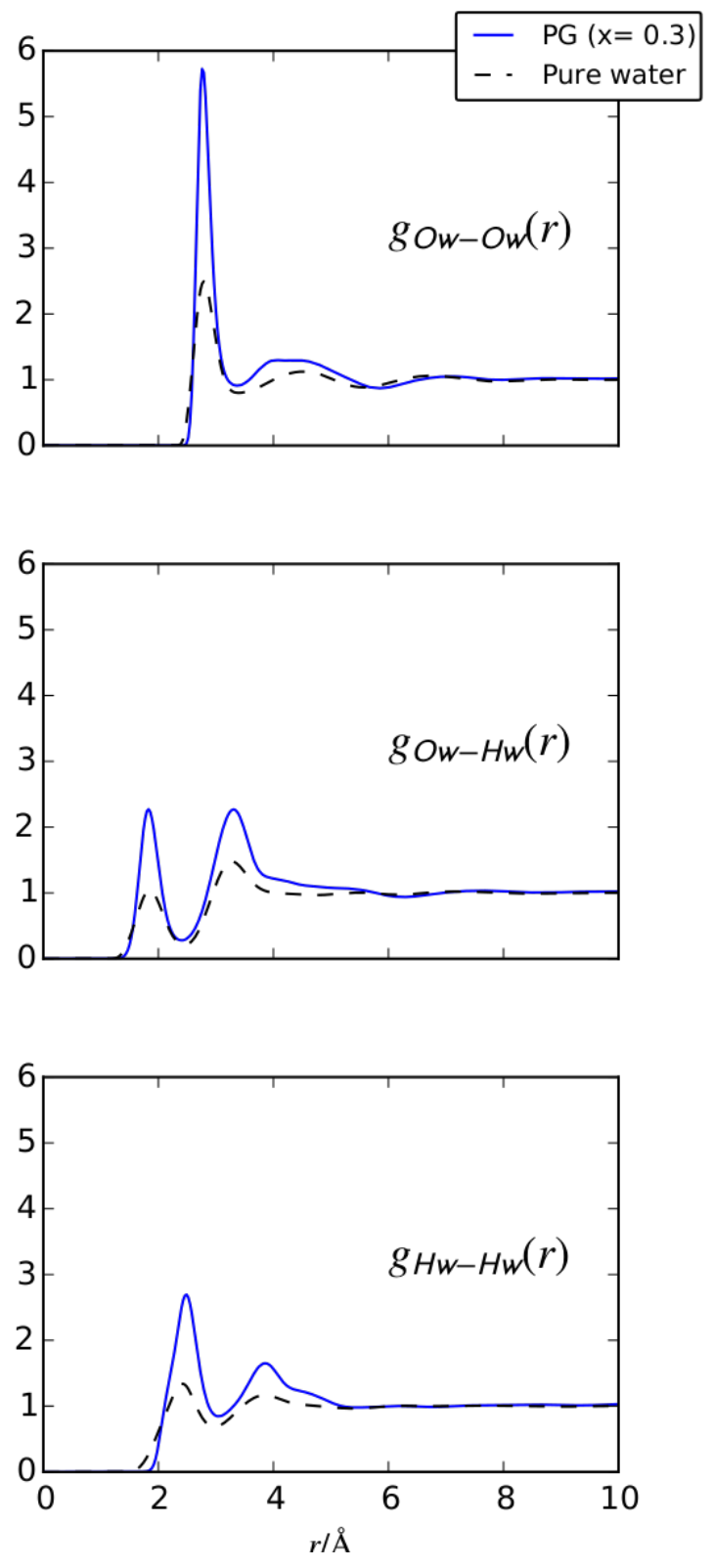

FIG. 3: The $g(r)$ s for water-water interactions in a $30 \mathrm{~mol} \%$ aqueous propylene glycol solution (blue) and pure water (black).

PG hydroxyl groups for the R-enantimomer with water are shown in Fig. 4, the S-enantiomer shows virtually identical $g(r)$ s for these functions and these are included in the Supplementary Information. From this figure there is a direct hydrogen bond from the water hydrogen to both both $\mathrm{O} 1$ and $\mathrm{O} 2$ groups and between the PG hydroxyl hydrogen atoms with the water Ow. All of these $g(r)$ s have a peak distance of $1.86 \AA$, which is indicative of a short, strong hydrogen bond. ${ }^{76}$ The location of nearest neighbor water molecules around the hydroxyl groups are shown in the 3-dimensions by the SDMs in Fig. 5 for $30 \%$ of those molecules classed as a nearest neighbor around each of the hydroxyl groups and the cut-throughs show 
TABLE III: Coordination numbers for the first shell of the water-water $g(r)$ s.

\begin{tabular}{ccc}
\hline \hline$g_{\alpha \beta}(r)$ & $n_{\alpha}^{\beta}$ & $r_{2}(\AA)$ \\
\hline$O w-O w$ & 2.5 & 3.39 \\
$O w-H w$ & 1.1 & 2.40 \\
$H w-H w$ & 3.2 & 3.04 \\
\hline \hline
\end{tabular}

TABLE IV: Coordination numbers for the first shell of the hydroxyl-water $g(r)$ s.

\begin{tabular}{ccc}
\hline \hline$g_{\alpha \beta}(r)$ & $\left(n_{\alpha}^{\beta}\right)$ & $r_{2}(\AA)$ \\
\hline$O 1-O w$ & 1.6 & 3.60 \\
$O 2-O w$ & 1.6 & 3.60 \\
$O 1-H w$ & 0.7 & 2.47 \\
$O 2-H w$ & 0.7 & 2.47 \\
$H 1-O w$ & 0.4 & 2.50 \\
$H 2-O w$ & 0.4 & 2.50 \\
\hline \hline
\end{tabular}

the absolute density of the water molecules around the hydroxyl groups. Both SDMs show two nearest neighbor water locations - where for one water is accepting a hydrogen bond from the - $\mathrm{OH}$ group and the other shows the nearest neighbor water molecules acting as hydrogen bond donors, below the hydroxyl oxygen. For the second hydroxyl group - located distally on the molecule (Fig. 2) - the density cloud is suggestive of less molecules being present within this shell compared with the central (Or2) hydroxyl group. However both the cut-throughs and the coordination numbers for the $g(r) \mathrm{s}$ in Table IV show a similar number of molecules within this nearest neighbor shell.

In order to probe whether self-self interactions between propylene glycol molecules are prevalent over propylene glycol-water interactions, a preferential binding coefficient, $B(r)$, which removes any dependence on concentration, can be determined. For a given group on a central propylene glycol that could preferentially bind to water $(w)$ or other propylene glycol molecules $(p)$ within a given distance range, $r, B(r)$ can be measured using the following relation;

$$
B_{p}(r)=n_{p}^{p}(0, r)-\frac{c_{p}}{c_{w}} n_{p}^{w}(0, r)
$$

where $n_{p}^{w}$ signifies the coordination number for water around propylene glycol and $c_{p}$ and $c_{w}$ are the relative atomic concentrations of the chosen atoms for propylene glycol and water, respectively. The value of $B(r)$ will be negative if propylene glycol is preferentially hydrated by water, whilst it will be positive if it is more likely to self-associate. $B(r)$ is linked to the Kirkwood-Buff theory of liquids ${ }^{77}$ and has been used to characterise the preferential binding of chloride ions versus water to the tripeptide glycyl-propyl-glycinamide. ${ }^{72}$

Fig. 6 shows the affinity of each hydroxyl oxygen to bind to a hydroxyl hydrogen of another PG molecule ver-
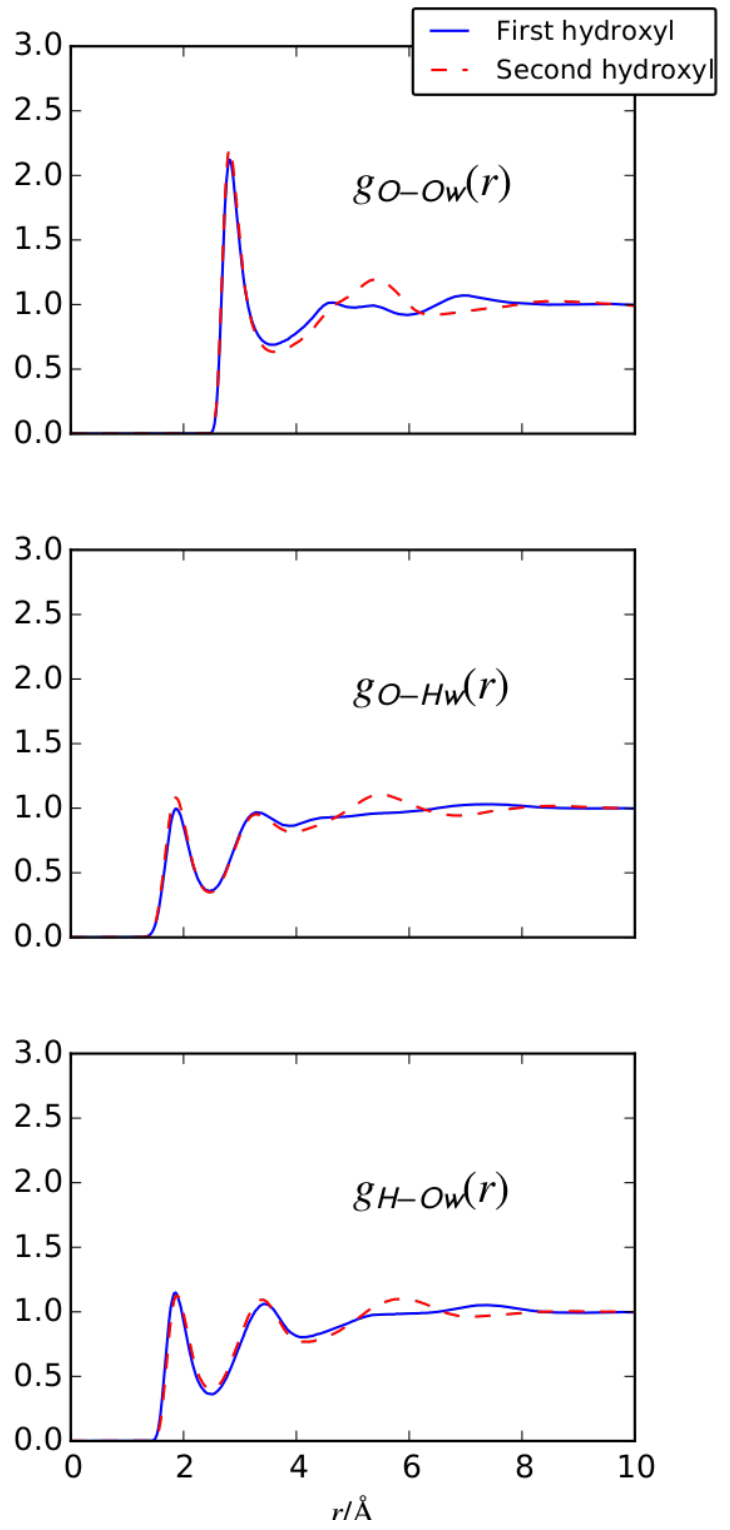

FIG. 4: The $g(r)$ s for interactions of the first (blue) and second (red) hydroxyl groups of the R-propylene glycol enantimomer with water in a $30 \mathrm{~mol} \%$ aqueous propylene glycol solution.

sus a water hydrogen, using $B(r)$ over a distance range of $10 \AA$. In the nearest neighbor distance range, below $\sim 3 \AA$, the $B(r)$ curves are relatively flat, signifying no clear preference of the PG hydroxyl groups to bind to either water or other PG molecules. However, at distance ranges greater than around $4 \AA$ all of the propylene glycol hydroxyl groups show a clear preference to interact with water rather than through self-self electrostatic interactions. This is likely due, in part, to the fact there are a larger number of water molecules compared with propylene glycol molecules in the solution but also suggests that there is an absence of large scale aggregation between propylene glycol molecules by virtue of their hy- 


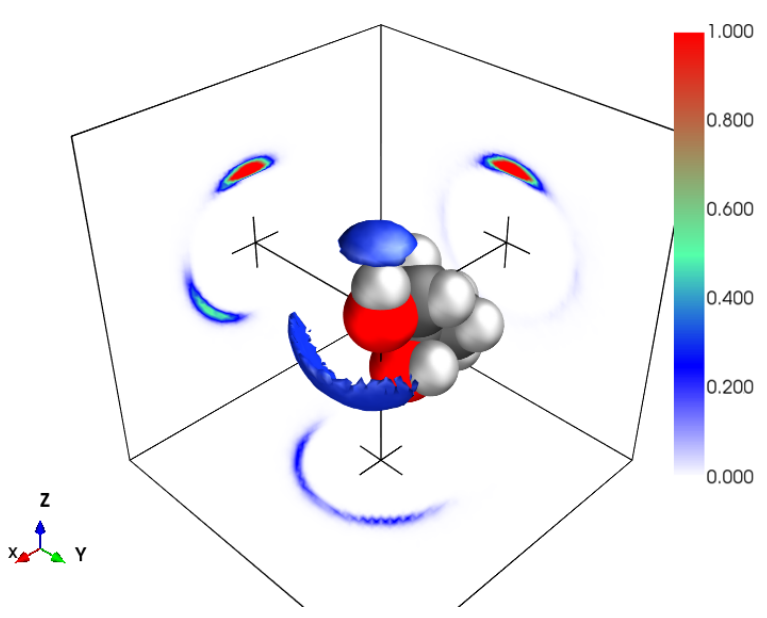

(a)

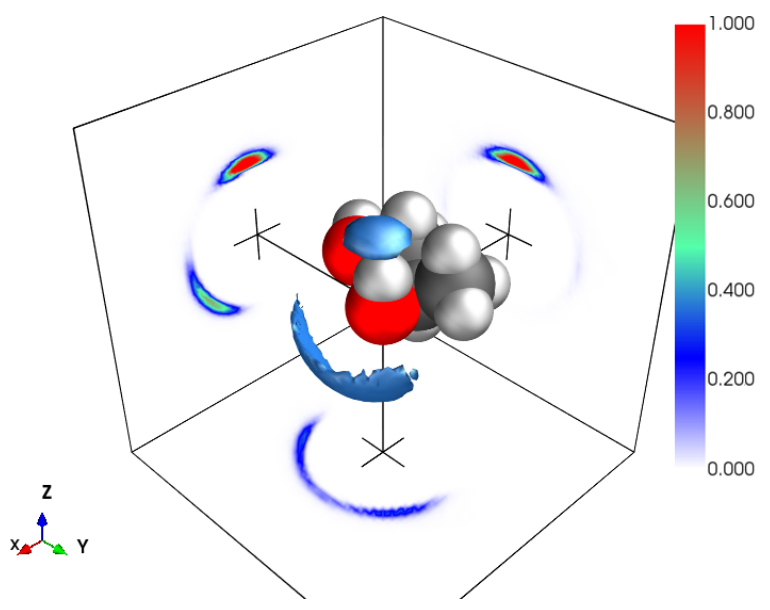

(b)

FIG. 5: SDMs showing the nearest neighbor analyses for the hydration of the (a) first and (b) second hydroxyl groups for R-propylene glycol. The isopycnic surface represents the location of $30 \%$ of the water molecules considered a nearest neighbour contact. The scale bar shows the density of nearest neighbors around a central propylene glycol in $\AA^{3}$

droxyl groups.

Additionally, Fig. 6 (3rd column) shows the $B(r)$ curve for PG self-interactions via the hydroxyl group, where these curves suggest a distance dependent interaction, where at larger distances a PG hydroxyl is more likely to bind to the central hydroxyl hydrogen, $\mathrm{H} 2$, whilst $\mathrm{O} 1$ is preferred in shorter distance ranges. Relating this back to Fig. 5, the preference for propylene glycol interactions with the first hydroxyl could explain why there is a subtle decrease in hydration of this group.

\section{Hydrophobic hydration}

Figure 7 shows the interactions between water and $\mathrm{PG}$ methylene atoms. Despite being a typical 'hydropho-
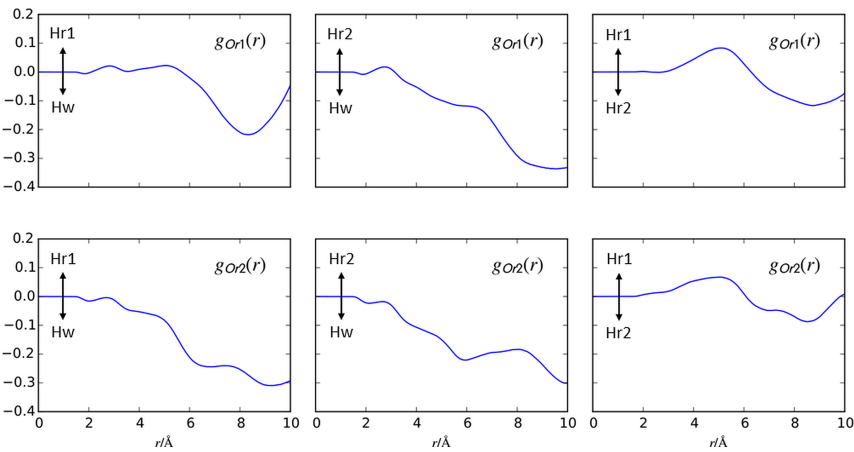

FIG. 6: The preferential binding coefficient, $B(r)$, for both $-\mathrm{OH}$ groups for water versus proplyene glycol.
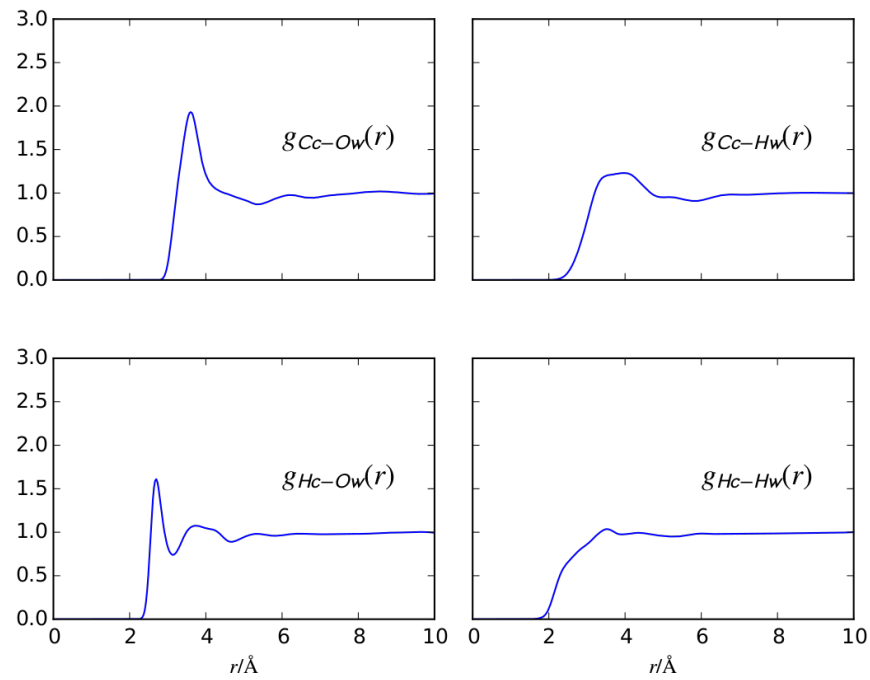

FIG. 7: The $g(r)$ s for methyl-water interactions in a $30 \mathrm{~mol} \%$ aqueous propylene glycol solution.

bic' group, the $g(r)$ for Hc-Ow shows a fairly significant first coordination shell interaction at $2.70 \AA$ between the methyl hydrogen and the surrounding water solvent, which is within a distance range to be considered a weak hydrogen bonding motif. ${ }^{76}$ As a comparison, whilst aqueous tertiary butanol (mole fraction between $0.06-0.16)^{36}$ shares a similar methyl carbon - water first coordination shell peak, it does not have the distinct hydration interaction shell seen for methyl hydrogen atoms in Fig. 7. The coordination numbers for these functions in Table V, shows that each $-\mathrm{CH}_{3}$ hydrogen atom is, on the average, coordinated to 0.8 water oxygens. This value is comparable to those observed previously for hydrogen bonds between the PG hydroxyl groups and water molecules (Table IV). The extent that hydration is observed here could, in part, be a consequence of the starting model, where for the current set of potentials there is a charge of -0.273 on the methyl carbon, and the Hc-Ow correlation is only around $3-5 \%$ of the scattering signal in most of the measured data sets.

What is unclear from the one-dimensional $g(r)$ is whether this hydrogen bonding interaction is in fact due 
TABLE V: Coordination numbers for the first shell of the alkyl-water $g(r)$ s shown in Fig. 7 .

\begin{tabular}{ccc}
\hline \hline$g_{\alpha \beta}(r)$ & $\left(n_{\alpha}^{\beta}\right)$ & $r_{2}(\AA)$ \\
\hline$C c-O w$ & 2.7 & 4.00 \\
$H c-O w$ & 0.8 & 3.12 \\
\hline \hline
\end{tabular}

to a Hc-Ow hydrogen bond or is merely due to the presence of water hydrogen bonding to the central -OH group of $\mathrm{PG}$. The SDM for the nearest neighboring water molecules around the $-\mathrm{CH}_{3}$ group (Fig. 8) shows that the closest contacts between the methyl group and water are not by virtue of direct $\mathrm{Hm}-\mathrm{Ow}$ hydrogen bonds but rather show that the nearest neighbor density is located between the methyl hydrogens. This is potentially a result of the charge on the central carbon atom and it may be that the methyl carbon forms an electrostatic interaction with the $\mathrm{Ow}$ water atoms. This would also account for the fact that the $\mathrm{Cc}-\mathrm{Hw} g(r)$ is relatively flat compared with the $\mathrm{Cc}-\mathrm{Ow} g(r)$, that is these nearest neighbor waters are fairly disoriented with respect to the $\mathrm{Cc}-\mathrm{Hw}$ interaction. Moreover this $\mathrm{Cc}-\mathrm{Ow}$ contact would allow for a more direct hydrogen bonding interaction between $\mathrm{Ow}$ and the Hc methyl hydrogen. That the $\mathrm{Cc}-\mathrm{Ow}$ interactions are more ordered is also supported by the presence of a more highly localized first nearest neighbor density in the cut-throughs of the SDM (Fig. 8) in the $+y$-direction, directly in front of the methyl Cc atom. This suggests that there is tangential orientation of water around the methyl group, similar to the hydrophobic hydration of methane in water. ${ }^{78}$

The presence of hydrophobic hydration is not an uncommon phenomenon, where it is the hydration of such alkyl groups that influences the stability with varying temperature for proteins. ${ }^{20}$ Previous neutron diffraction studies have elicited the occurrence of hydration of hydrophobic groups in molecules such as lipids, ${ }^{79,80}$ amino acids $^{59}$ and neurotransmitters, ${ }^{60}$ which are potentially influenced by the electrostatics of a nearby polar group. For example, research by Foglia et al in the $\mathrm{C}_{3}$-PC lipid revealed that the hydrogen atoms methylene group adjacent to the $\mathrm{N}\left(\mathrm{CH}_{3}\right)_{3}$ were also able to coordinate approximately 1 water molecule each. ${ }^{79}$

\section{Propylene glycol association}

While the $B(r)$ curves do not show any large-scale self association of propylene glycol molecules, these molecules will likely still have some degree of self association through hydroxyl group interactions. The $g(r) \mathrm{s}$ for the $\mathrm{O} \cdots \mathrm{H}$ interactions between the $-\mathrm{OH}$ groups on $\mathrm{R}$ enantiomers for propylene glycol are shown in Fig.9 (see Fig. SI.6 for interactions for other enantiomer combinations), where direct hydrogen bonding interactions can be found between hydroxyl oxygen atoms and the $-\mathrm{OH}$

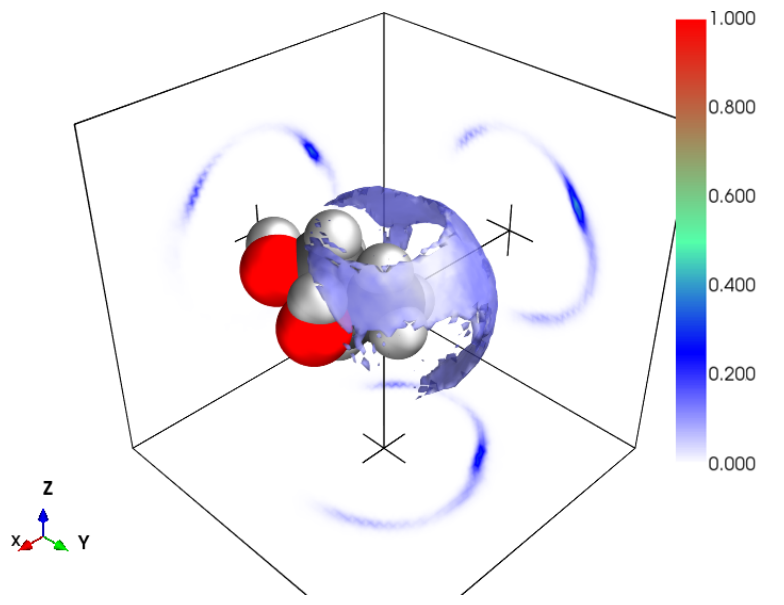

FIG. 8: SDMs showing the nearest neighbour analyses for the hydration of the methyl group. The isopycnic surface represents the location of $30 \%$ of the water molecules considered a nearest neighbour contact. The scale bar shows the density of nearest neighbors around a central propylene glycol in $\AA^{3}$
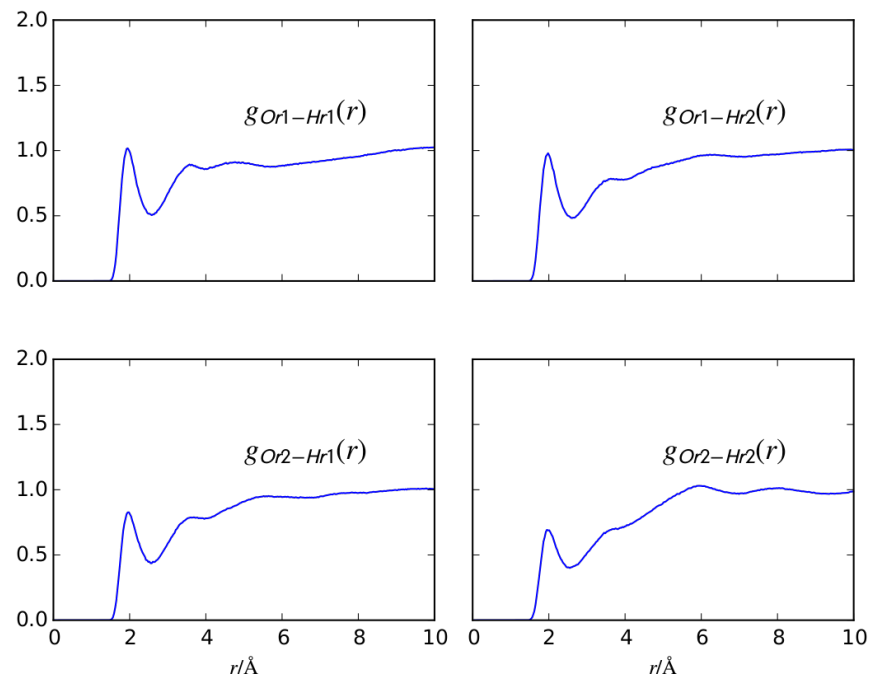

FIG. 9: The $g(r)$ s for propylene glycol -OH self interactions in a $30 \mathrm{~mol} \%$ aqueous propylene glycol solution.

hydrogens on adjacent propylene glycol molecules at distances of around $1.95 \AA$. The coordination numbers for these functions (Table VI), regardless of which hydroxyl group or enantiomer is 0.4 , which is lower than the coordination observed for $-\mathrm{OH}$ interactions with water. A clustering analysis, used to determine the size of any clusters within the solution, showed that through all hydroxyl group interactions only clusters of up to 10 molecules could be formed (Fig. SI.9a), with a less than 1\% probability of clusters with 3 or more molecules.

Fig. 10 shows the SDMs of the interactions between both hydroxyls for the R-enantiomer. These show $10 \%$ of the methyl groups considered nearest neighbor con- 
TABLE VI: Coordination numbers for the first shell of the hydrophilic propylene glycol - propylene glycol interactions. The numbers correspond to the values attained for all enantiomer combinations; R-R, R-S, S-R and S-S.

\begin{tabular}{lcc}
\hline \hline$g_{\alpha \beta}(r)$ & $\left(n_{\alpha}^{\beta}\right)$ & $r_{2}(\AA)$ \\
\hline$O-O$ & 0.4 & 3.65 \\
$O-H$ & 0.1 & 2.60 \\
$H-O$ & 0.1 & 2.60 \\
\hline \hline
\end{tabular}

TABLE VII: Coordination numbers for the first shell of the alkyl-alkyl $g(r)$ s shown in Fig. 11.

\begin{tabular}{ccc}
\hline \hline$g_{\alpha \beta}(r)$ & $\left(n_{\alpha}^{\beta}\right)$ & $r_{2}(\AA)$ \\
\hline$C c-C c$ & 2.3 & 5.00 \\
$H c-H c$ & 1.2 & 3.15 \\
\hline \hline
\end{tabular}

tacts and a lower density range in the surrounding cloud for clarity. The equivalent plots for S- can be found in the Supplementary Information (Fig. SI.5). For each combination, it is clear that the molecule coordinates hydroxyl groups through the hydrogen, represented by density in the $+z$ direction, and the oxygen atoms, where the density for the latter is in the $+x$ direction. For the interactions between two R- enantiomers, the density is highly localised for O1-O2 and O2-O1 interactions, compared to the more diffuse distribution observed for O1-O1 and $\mathrm{O} 2-\mathrm{O} 2$ interactions. What is more interesting is that this localisation is no longer observed the same when the interaction is between an R- and S- enantiomer. Whilst interactions are possible for all combinations, this difference suggests that there could be a slight preference propylene glycol molecules to interact through certain enantiomer combinations. Although no change in coordination number is observed for each combination, the results for the SDMs may correlate to a small decrease in peak position for the interactions showing localisation. Indeed, the density levels in atoms $/ \AA^{3}$ for these isopycnic surfaces are below 0.1 , so the analyses completed here are only subtle differences between the interactions.

As well as hydrophilic interactions, it is also possible that association could occur through the hydrophobic groups between PG molecules. In fact, the Hc-Hc $g(r)$ shows a peak at $2.64 \AA$ (Fig. 11), with each atom coordinating just over 1 hydrogen from the $-\mathrm{CH}_{3}$ of another PG molecule. Related SDMs for the R-R and R-S methyl interaction are shown in Fig. 12, where the closest contacts are not over the hydrogen atoms, but in between them, similar to the hydration of the methyl groups shown in Fig. 8. The S-S and the S-R interactions are similar and are shown in the Supporting Information. However, whilst these interactions do show some clustering (Fig. SI.9a), the probability of this is extremely low, with hydration interactions generally preventing any extensive association from occurring.
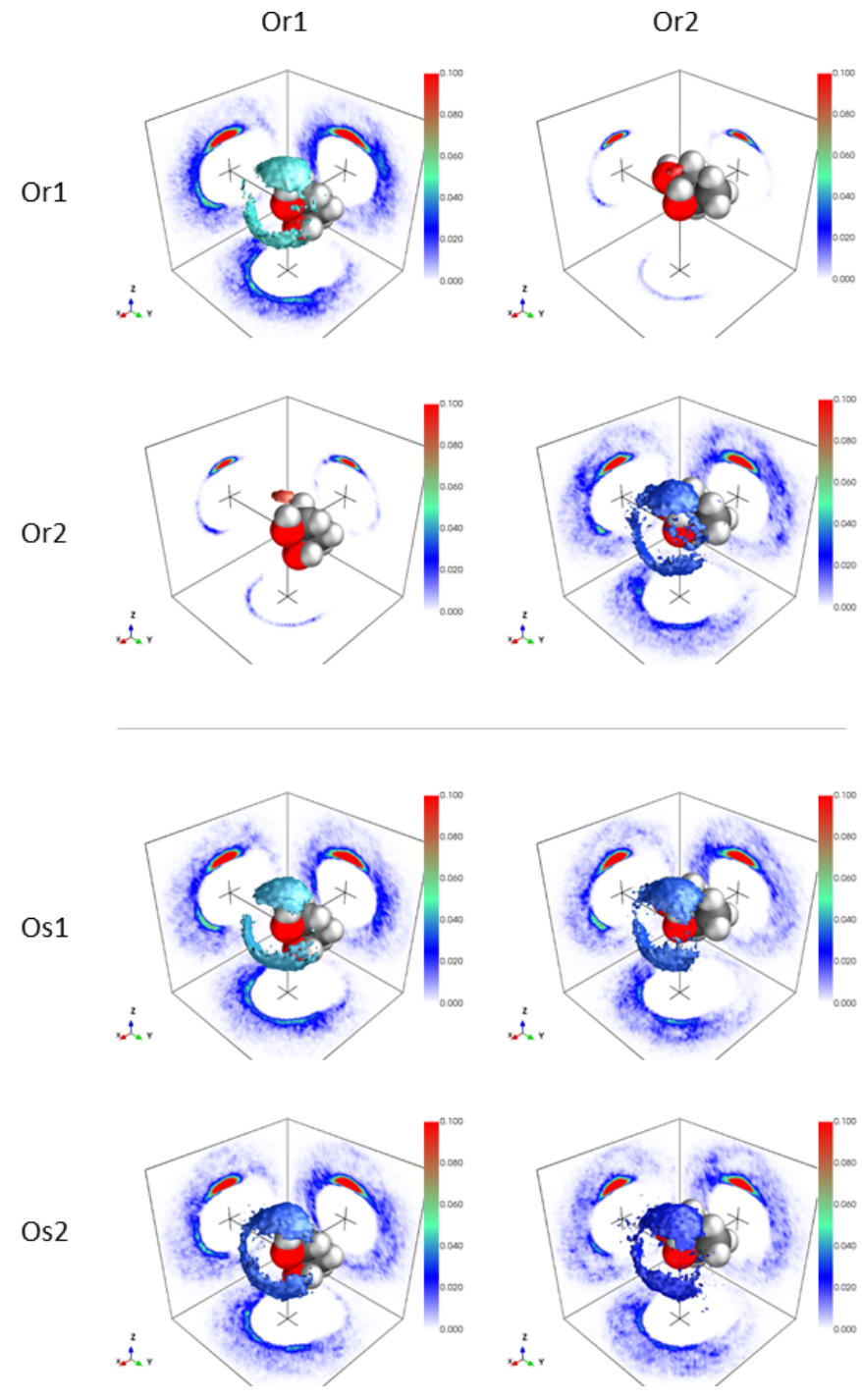

FIG. 10: SDMs showing the nearest neighbour analyses for the hydrophilic propylene glycol - propylene glycol

interactions. The isopycnic surface represents the location of $10 \%$ of the hydroxyl groups considered a nearest neighbour contact. The scale bar shows the density of nearest neighbors around a central propylene glycol in $\AA^{3}$

\section{CONCLUSIONS}

In the present aqueous PG solutions the water-water coordination observed suggest that the bulk water structure is largely intact, similar to the water structure observed in DMSO/water, ${ }^{14}$ methanol, ${ }^{13}$, glycerol $^{43}$ and sorbitol $^{38}$ solutions. A shift of the second peak in the Ow-Ow water $g(r)$ to lower $r$-values also occurs, similar to what for aqueous solutions of sorbitol ${ }^{38}$ and both methanol and glycerol solutions ${ }^{81}$ at low solute concentrations, and has been suggested to indicate a slightly compressed tetrahedral coordination of water as it is a similar effect observed in pure water under pressure. ${ }^{82}$ 

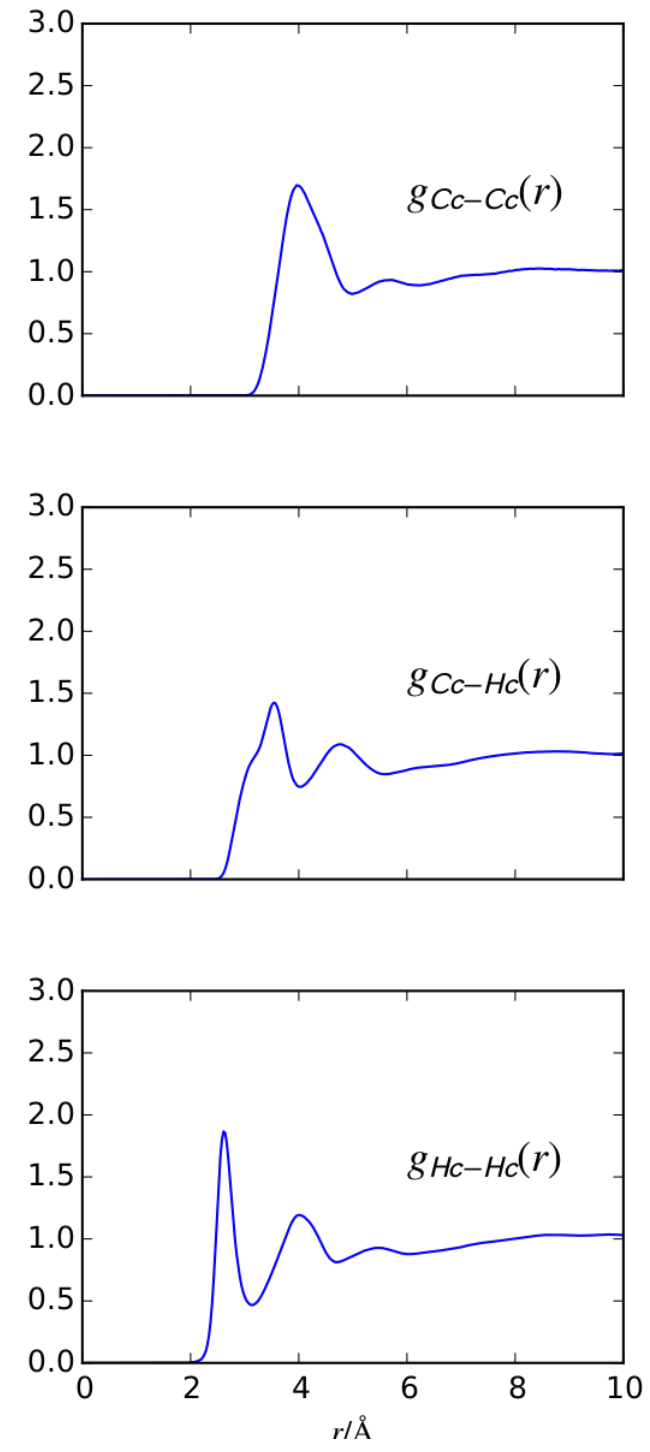

FIG. 11: The $g(r)$ s for methyl-methyl interactions in a $30 \mathrm{~mol} \%$ aqueous propylene glycol solution.

This change in the bulk water structure has been postulated to contribute to the mechanism by which cryoprotectants, such as PG, prevent ice formation. ${ }^{81}$

With two hydroxyl groups, the results here show that PG is readily hydrated by surrounding water molecules, through both hydrogen bond donation and acceptance, with the SDM plots in Fig. 5 showing the nearest neighbor water molecules in similar highly localized positions about both - $\mathrm{OH}$ groups. $\mathrm{PG}$ molecules can also clearly self-associate electrostatically via their hydroxyl groups where, for the most part, the PG nearest neighbor hydroxyl-hydroxyl interactions are similar to that of the hydration of both of these groups as can been seen in Fig.10. However, interestingly the hydroxyl-hydroxyl interactions seem to be more localised for the case where the first and second hydroxyl groups co-join when the enantiomer is the same (R-R and S-S), suggesting a sub-

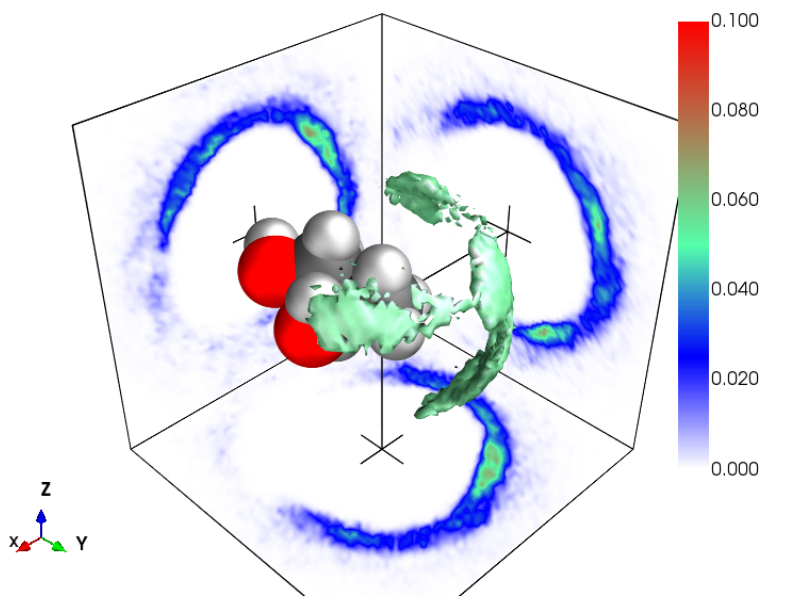

(a)

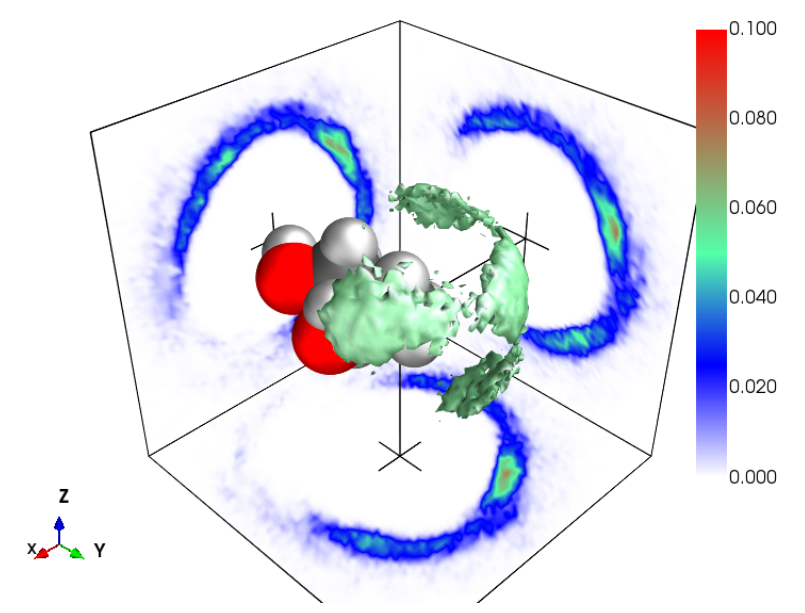

(b)

FIG. 12: SDMs showing the nearest neighbour analyses of methyl-methyl interactions for (a) R-R and (b) R-S molecules. The isopycnic surface represents the location of $10 \%$ of the methyl groups considered a nearest neighbour contact. The scale bar shows the density of nearest neighbors around a central propylene glycol in $\AA^{3}$

tle preference for certain interactions to take place between PG molecules. Using the preferential binding coefficient, it was determined that the hydration of both hydroxyl groups supersede those governing self-association, preventing the formation of larger-scale PG structures through these hydrophilic interactions.

In the present solution, the hydrophobic methyl group on PG also appears to form strong interactions with the surrounding water solvent as evidenced by a relatively sharp first peak in the Hc-Ow $g(r)$ (Fig. 8, albeit it at relatively long distances, $\sim 2.7 \AA$ ), where these water oxygen appear to be located between the hydrogens on the $-\mathrm{CH}_{3}$ group rather than as a result of a direct hydrogen bond. It should also be noted that the seed potential for the Cc atom on the PG methylene group carries a charge of -0.273 and as such the methyl-water interactions may, in 
part, be somewhat over-emphasized in the current EPSR model. Although there is a somewhat preferential interaction with the methyl group, this is not necessarily evidence for the increased ordering of water, as was similarly concluded for other alcohol solutions. ${ }^{83}$ Given that the nearest neighbor hydration shows a fairly delocalized cloud of density surrounding this group (Fig. 8). Moreover, that the water hydrogen atoms show a largely disordered interaction around that group showing that they are not participant in hydrogen bonding to this group also indicates that the methyl groups are not 'enhancing' the bulk water structure. The slight electrorestrictive effect on water, as evidenced by a shift in the second shell $\mathrm{Ow}-\mathrm{Ow}$ peak, is more likely to be a result of $\mathrm{OH}$-water interactions as has been observed previously for alcohols.

Interestingly, methyl-methyl interactions between PG molecules also occur at comparable locations and at similar radial distances as the methyl hydration interactions. Even with the presence of these methyl-methyl interactions the competing hydration levels still make the probability of the formation any large-scale PG structures extremely low.

The preference for hydration of the $-\mathrm{OH}$ groups over PG self-self interactions could still account for the tendency for propylene glycol solutions to remain amorphous, whereby its dispersion throughout the solution prevents formation of a continuous network of PG. In more complex systems, this preference may translate into an increase PG-water interactions, such as is thought to occur for DMSO in aqueous solutions containing lipids. ${ }^{84}$ In addition, this behavior may have implications for how PG acts to enhance the apparent solublity of compounds in lipid-containing systems. Recent simulations of lipid bilayers in aqueous propylene glycol have speculated that propylene glycol may form a surface over the lipid head groups to enhance penetration of pharmaceuticals ${ }^{20}$ and it may be that the preference of PG to be hydrated rather than associate with itself may also extend into a preference of PG to interact with lipids rather than either itself or the surrounding lipid environment.

\section{SUPPLEMENTARY INFORMATION}

Supplementary material, which has been referred to in the main text, can be found via the following link.

\section{ACKNOWLEDGMENTS}

We thank the ISIS Facility (Rutherford Appleton Laboratories, STFC, UK) for the allocation of neutron beam time, the UK Engineering and Physical Sciences Research Council for (EP/J002615/1) for funding.

\footnotetext{
${ }^{1}$ G. N. Bennett and K. Y. San, "Microbial formation, biotechnological production and applications of 1,2-propanediol." Applied microbiology and biotechnology 55, 1-9 (2001).
}

${ }^{2}$ A. Chauvel and G. G. Lefebvre, "Petrochemical Processes: Volume 2: Oxygenated, Chlorinated and Nitrated Derivatives." Gulf Publishing Company, 26 (1989).

${ }^{3}$ M. Pagliaro, R. Ciriminna, H. Kimura, M. Rossi, and C. DellaPina, "From Glycerol to Value-Added Products," Angewandte Chemie International Edition 46, 4434-4440 (2007).

${ }^{4}$ D. H. Rasmussen and A. P. MacKenzie, "Glass transition in amorphous water. Application of the measurements to problems arising in cryobiology," The Journal of Physical Chemistry $\mathbf{7 5}$ 967-973 (1971).

${ }^{5}$ J. M. Hutchinson and A. J. Kovacs, "A simple phenomenological approach to the thermal behavior of glasses during uniform heating or cooling," Journal of Polymer Science: Polymer Physics Edition 14, 1575-1590 (1976).

${ }^{6} \mathrm{P}$. Boutron and A. Kaufmann, "Stability of the amorphous state in the system water-1,2-Propanediol," Cryobiology 16, 557-568 (1979).

${ }^{7}$ O. Kirichek, A. K. Soper, B. Dzyuba, S. K. Callear, and B. Fuller, "Strong isotope effects on melting dynamics and ice crystallisation processes in cryo vitrification solutions." PLoS ONE 10, e0120611 (2015).

${ }^{8}$ C. A. Amorim, D. Rondina, A. P. R. Rodrigues, P. B. D. Gonçalves, J. R. de Figueiredo, and A. Giorgetti, "Cryopreservation of isolated ovine primordial follicles with propylene glycol and glycerol," Fertility and Sterility 81, 735-740 (2004).

${ }^{9}$ L. A. Marquez-Curtis, A. Janowska-Wieczorek, L. E. McGann, and J. A. Elliott, "Mesenchymal stromal cells derived from various tissues: Biological, clinical and cryopreservation aspects," Cryobiology 71, 181-197 (2015).

${ }^{10}$ H. Newton, J. Fisher, J. R. Arnold, D. E. Pegg, M. J. Faddy, and R. G. Gosden, "Permeation of human ovarian tissue with cryoprotective agents in preparation for cryopreservation." $\mathrm{Hu}-$ man reproduction (Oxford, England) 13, 376-380 (1998).

${ }^{11}$ O. Kirichek, A. K. Soper, B. Dzyuba, and W. V. Holt, "Segregated water observed in a putative fish embryo cryopreservative," Royal Society Open Science 3, 150655 (2016).

${ }^{12}$ L. Dougan, S. P. Bates, R. Hargreaves, J. P. Fox, J. Crain, J. L. Finney, V. Reat, and A. K. Soper, "Methanol-water solutions: A bi-percolating liquid mixture," Journal of Chemical Physics 121, 6456-6462 (2004).

${ }^{13}$ S. Dixit, A. K. Soper, J. L. Finney, and J. Crain, "Water structure and solute association in dilute aqueous methanol," Europhysics Letters 59, 377-383 (2002).

${ }^{14}$ S. E. McLain, A. K. Soper, and A. Luzar, "Investigations on the structure of dimethyl sulfoxide and acetone in aqueous solution," The Journal of Chemical Physics 127, 174515 (2007).

${ }^{15}$ A. K. Soper and A. Luzar, "Orientation of water molecules around small polar and nonpolar groups in solution: a neutron diffraction and computer simulation study," The Journal of Physical Chemistry 100, 1357-1367 (1996).

${ }^{16}$ A. C. Williams and B. W. Barry, "Penetration enhancers." Advanced drug delivery reviews 56, 603-618 (2004).

${ }^{17}$ S. Soltanpour, Z. Bastami, S. Sadeghilar, M. Kouhestani, F. Pouya, and A. Jouyban, "Solubility of Clonazepam and Diazepam in Polyethylene Glycol 200, Propylene Glycol, N-Methyl Pyrrolidone, Ethanol, and Water at (298.2 to 318.2) K and in Binary and Ternary Mixtures of Polyethylene Glycol 200, Propylene Glycol, and Water at 298.2 K," Journal of Chemical and Engineering Data 58, 307-314 (2013).

${ }^{18} \mathrm{H}$. A. Benson, "Transdermal drug delivery: penetration enhancement techniques," Current Drug Delivery 2, 23-33 (2005).

${ }^{19}$ Z. E. Hughes, C. J. Malajczuk, and R. L. Mancera, "The Effects of Cryosolvents on DOPC- $\beta$-Sitosterol Bilayers Determined from Molecular Dynamics Simulations," The Journal of Physical Chemistry B , 2617-2624 (2013).

${ }^{20}$ C. J. Malajczuk, Z. E. Hughes, and R. L. Mancera, "Molecular dynamics simulations of the interactions of DMSO, mono- and polyhydroxylated cryosolvents with a hydrated phospholipid bilayer." Biochimica et Biophysica Acta 1828, 2041-2055 (2013). 
${ }^{21}$ R. D. Harvey, N. Ara, R. K. Heenan, D. J. Barlow, P. J. Quinn, and M. J. Lawrence, "Stabilization of distearoylphosphatidylcholine lamellar phases in propylene glycol using cholesterol." Molecular pharmaceutics 10, 4408-4417 (2013).

${ }^{22}$ M. M. A. Elsayed, O. Y. Abdallah, V. F. Naggar, and N. M. Khalafallah, "PG-liposomes: novel lipid vesicles for skin delivery of drugs," Journal of Pharmacy and Pharmacology 59, 14471450 (2007).

${ }^{23}$ K.-C. Kang, C.-I. Lee, H.-B. Pyo, and N.-H. Jeong, "Preparation and Characterization of Nano-Liposomes Using Phosphatidylcholine," Journal of Industrial and Engineering Chemistry 11, 847-851 (2005).

${ }^{24}$ R. M. Watkinson, R. H. Guy, J. Hadgraft, and M. E. Lane, "Optimisation of cosolvent concentration for topical drug delivery ii: Influence of propylene glycol on ibuprofen permeation," Skin Pharmacol. Physiol. 22, 225-230 (2009).

${ }^{25}$ V. B. Junyaprasert, P. Singhsa, J. Suksiriworapong, and D. Chantasart, "Physicochemical properties and skin permeation of span 60/tween 60 niosomes of ellagic acid," International Journal of Pharmaceutics 423, 303 - 311 (2012).

${ }^{26}$ T.-H. Jeong and S.-G. Oh, "Lyotropic behaviors of a phospholipid-based lamella liquid crystalline phase hydrated by propylene glycol as a polar solvent: Correlation of dspc vs pg concentration," Bull. Kor. Chem. Soc. 28, 208-114 (2007).

${ }^{27}$ Z. E. Hughes and R. L. Mancera, "Molecular Mechanism of the Synergistic Effects of Vitrification Solutions on the Stability of Phospholipid Bilayers," Biophysical Journal 117, 3362-3375 (2014).

${ }^{28}$ A. V. Gubskaya and P. G. Kusalik, "Molecular Dynamics Simulation Study of Ethylene Glycol, Ethylenediamine, and 2Aminoethanol. 1. The Local Structure in Pure Liquids," The Journal of Physical Chemistry A 108, 7151-7164 (2004).

${ }^{29}$ L. Saiz, J. A. Padro, and E. Guardia, The Journal of Chemical Physics 114, 3187 (2001).

${ }^{30}$ A. Kaiser, O. Ismailova, A. Koskela, S. E. Huber, M. Ritter, B. Cosenza, W. Benger, R. Nazmutdinov, and M. Probst, "Ethylene glycol revisited: Molecular dynamics simulations and visualization of the liquid and its hydrogen-bond network." Journal of Molecular Liquids 189, 20-29 (2014).

${ }^{31}$ A. K. Soper and A. Luzar, "Orientation of Water Molecules around Small Polar and Nonpolar Groups in Solution: A Neutron Diffraction and Computer Simulation Study," The Journal of Physical Chemistry 100, 1357-1367 (1996).

${ }^{32}$ A. K. Soper, "GudrunN and GudrunX: Programs for Correcting Raw Neutron and X-ray Diffraction Data to Differential Scattering Cross Section," RAL Report RAL-TR-201 (2011).

${ }^{33}$ V. F. Sears, "Neutron scattering lengths and cross sections," Neutron News 3, 26-37 (1992).

${ }^{34}$ A. K. Soper, "Empirical potential Monte Carlo simulation of fluid structure," Chemical Physics 202, 295-306 (1996).

${ }^{35}$ A. K. Soper, "Partial structure factors from disordered materials diffraction data: An approach using empirical potential structure refinement," Physical Review B 72, 104204-104215 (2005).

${ }^{36}$ D. T. Bowron, J. L. Finney, and A. K. Soper, "Structural Investigation of Solute - Solute Interactions in Aqueous Solutions of Tertiary Butanol," The Journal of Physical Chemistry B 102, 3551-3563 (1998).

${ }^{37}$ D. T. Bowron, A. K. Soper, and J. L. Finney, "Temperature dependence of the structure of a 0.06 mole fraction tertiary butanolwater solution," The Journal of Chemical Physics 114, 6203 (2001).

${ }^{38}$ S. G. Chou, A. K. Soper, S. Khodadadi, J. E. Curtis, S. Krueger, M. T. Cicerone, A. N. Fitch, and E. Y. Shalaev, "Pronounced Microheterogeneity in a Sorbitol-Water Mixture Observed through Variable Temperature Neutron Scattering," The Journal of Physical Chemistry B 116, 4439-4447 (2012).

${ }^{39} \mathrm{~S}$. Imberti and D. Bowron, "Formic and Acetic Acid Aggregation in the Liquid State," Journal of Physics: Condensed Matter 22, 404212 (2010).
${ }^{40}$ F. Meersman, D. Bowron, A. K. Soper, and M. H. J. Koch, "Counteraction of Urea by Trimethylamine N-Oxide Is Due to Direct Interaction," Biophysical Journal 97, 2559-2566 (2009).

${ }^{41}$ J. J. Shephard, A. K. Soper, S. K. Callear, S. Imberti, J. S. O. Evans, and C. G. Salzmann, "Polar stacking of molecules in liquid chloroform." Chemical Communications 51, 4770-4773 (2015).

42 J. J. Towey, A. K. Soper, and L. Dougan, "Molecular Insight into the Hydrogen Bonding and Micro-segregation of a Cryoprotectant Molecule," The Journal of Physical Chemistry B 116, 13898-13904 (2012).

${ }^{43}$ J. J. Towey, A. K. Soper, and L. Dougan, "What Happens to the Structure of Water in Cryoprotectant Solutions?" Faraday Discussions 167, 159-176 (2013).

${ }^{44}$ P. Sillrén, J. Swenson, J. Mattsson, D. Bowron, and A. Matic, "The temperature dependent structure of liquid 1-propanol as studied by neutron diffraction and EPSR simulations." The Journal of Chemical Physics 138, 214501 (2013).

${ }^{45}$ T. F. Headen, C. A. Howard, N. T. Skipper, M. A. Wilkinson, D. T. Bowron, and A. K. Soper, "Structure of pi-pi interactions in aromatic liquids." Journal of the American Chemical Society 132, 5735-5742 (2010).

${ }^{46}$ J. A. McCune, A. H. Turner, F. Coleman, C. M. White, S. K. Callear, T. G. A. Youngs, M. Swadzba-Kwasny, and J. D. Holbrey, "Association and liquid structure of pyridine-acetic acid mixtures determined from neutron scattering using a 'free proton' epsr simulation model," Physical Chemistry Chemical Physics 17, 6767-6777 (2015).

${ }^{47}$ S. Busch, C. D. Bruce, C. Redfield, C. D. Lorenz, and S. E. McLain, "Water mediation is essential to nucleation of $\beta$-turn formation in peptide folding motifs." Angewandte Chemie (International ed. in English) 52, 13091-13095 (2013).

${ }^{48}$ S. Busch, C. D. Lorenz, J. Taylor, L. C. Pardo, and S. E. McLain, "Short-Range Interactions of Concentrated Proline in Aqueous Solution." The Journal of Physical Chemistry B 118, 1426714277 (2014).

${ }^{49}$ R. J. Gillams, C. D. Lorenz, and S. E. McLain, "Comparative atomic-scale hydration of the ceramide and phosphocholine headgroup in solution and bilayer environments," The Journal of Chemical Physics 144, 225101 (2016).

${ }^{50}$ A. J. Johnston, S. Busch, L. C. Pardo, S. K. Callear, P. C. Biggin, and S. E. McLain, "On the atomic structure of cocaine in solution," Physical Chemistry Chemical Physics 18, 991-999 (2016).

${ }^{51}$ W. B. O'Dell, D. C. Baker, and S. E. McLain, "Structural evidence for inter-residue hydrogen bonding observed for cellobiose in aqueous solution," PLoS ONE 7, e45311 (2012).

${ }^{52}$ S. E. McLain, A. K. Soper, and A. Watts, "Water structure around dipeptides in aqueous solutions." European Biophysics Journal 37, 647-655 (2008).

${ }^{53}$ S. E. McLain, A. K. Soper, A. E. Terry, and A. Watts, "Structure and hydration of L-proline in aqueous solutions." The Journal of Physical Chemistry B 111, 4568-4580 (2007).

54 "Water-peptide site-specific interactions: A structural study on the hydration of glutathione," Biophysical Journal 106.

${ }^{55}$ J. J. Shephard, S. K. Callear, S. Imberti, J. S. O. Evans, and C. G. Salzmann, "Microstructures of negative and positive azeotropes," Physical Chemistry Chemical Physics 18, 1922719235 (2016).

${ }^{56}$ E. J. Nilsson, V. Alfredsson, D. T. Bowron, and K. J. Edler, "A neutron scattering and modelling study of aqueous solutions of tetramethylammonium and tetrapropylammonium bromide," Physical Chemistry Chemical Physics 18, 11193-11201 (2016).

${ }^{57}$ N. Steinke, R. J. Gillams, L. C. Pardo, C. D. Lorenz, and S. E. McLain, "Atomic scale insights into ureapeptide interactions in solution," Physical Chemistry Chemical Physics 18, 3862-3870 (2016).

${ }^{58}$ N. H. Rhys, A. K. Soper, and L. Dougan, "The HydrogenBonding Ability of the Amino Acid Glutamine Revealed by Neutron Diffraction Experiments." The Journal of Physical Chem- 
istry B 116, 13308-13319 (2012).

${ }^{59}$ N. H. Rhys, A. K. Soper, and L. Dougan, "Hydrophilic association in a dilute glutamine solution is persistent with increasing temperature," The Journal of Physical Chemistry B 119, 1564415651 (2015)

${ }^{60}$ E. C. Hulme, A. K. Soper, S. E. McLain, and J. L. Finney, "The hydration of the neurotransmitter acetylcholine in aqueous solution." Biophysical Journal 91, 2371-2380 (2006).

${ }^{61}$ A. Sridhar, A. J. Johnston, L. Varathan, S. E. McLain, and P. C. Biggin, "The solvation structure of Alprazolam," Physical Chemistry Chemical Physics 18, 22416-22425 (2016).

${ }^{62}$ S. K. Callear, A. Johnston, S. E. McLain, and S. Imberti, "Conformation and interactions of dopamine hydrochloride in solution," The Journal of Chemical Physics" 142, 014502 (2015).

${ }^{63}$ C. Hardacre, J. D. Holbrey, S. E. J. McMath, D. T. Bowron, and A. K. Soper, "Structure of molten 1,3-dimethylimidazolium chloride using neutron diffraction," The Journal of Chemical Physics 118, 273-278 (2003).

${ }^{64}$ R. Mancinelli, A. Botti, F. Bruni, M. A. Ricci, and A. K. Soper, "Perturbation of water structure due to monovalent ions in solution." Physical Chemistry Chemical Physics 9, 2959-2967 (2007).

${ }^{65}$ D. T. Bowron, E. C. Beret, E. Martin-Zamora, A. K. Soper, and E. S. Marcos, "Axial structure of the Pd(II) aqua ion in solution." Journal of the American Chemical Society 134, 962-967 (2012).

${ }^{66}$ R. Hayes, S. Imberti, G. G. Warr, and R. Atkin, "Amphiphilicity determines nanostructure in protic ionic liquids," Physical Chemistry Chemical Physics 13, 3237-3247 (2011).

${ }^{67} \mathrm{~K}$. Vanommeslaeghe and A. D. MacKerell, "Automation of the CHARMM General Force Field (CGenFF) I: bond perception and atom typing." Journal of chemical information and modeling 52, 3144-54 (2012).

${ }^{68}$ M. D. Hanwell, D. E. Curtis, D. C. Lonie, T. Vandermeersch, E. Zurek, and G. R. Hutchison, "Avogadro: an advanced semantic chemical editor, visualization, and analysis platform," Journal of Cheminformatics 4, 17 (2012).

${ }^{69}$ H. J. C. Berendsen, J. R. Grigera, and T. P. Straatsma, "The missing term in effective pair potentials," The Journal of Physical Chemistry 91, 6269-6271 (1987).

${ }^{70}$ A. K. Soper, "Tests of the empirical potential structure refinement method and a new method of application to neutron diffraction data on water," Molecular Physics 99, 1503-1516 (2001).

71 "https://gcm.upc.edu/en/members/luiscarlos/angula/angula,".
${ }^{72}$ S. Busch, L. C. Pardo, W. B. O'Dell, C. D. Bruce, C. D. Lorenz, and S. E. McLain, "On the structure of water and chloride ion interactions with a peptide backbone in solution." Physical Chemistry Chemical Physics 15, 21023-21033 (2013).

${ }^{73}$ A. K. Soper, "The Radial Distribution Functions of Water as Derived from Radiation Total Scattering Experiments: Is There Anything We Can Say for Sure?" ISRN Physical Chemistry 2013 (2013).

${ }^{74}$ A. K. Soper, "The excluded volume effect in confined fluids and liquid mixtures," Journal of Physics: Condensed Matter 9, 2399 (1997).

${ }^{75}$ P. E. Mason and J. W. Brady, "Tetrahedrality and the relationship between collective structure and radial distribution functions in liquid water," The Journal of Physical Chemistry B 111, 5669-5679 (2007).

${ }^{76}$ T. Steiner, "The Hydrogen Bond in the Solid State," Angewandte Chemie International Edition 41, 48-76 (2002).

${ }^{77}$ J. G. Kirkwood and F. P. Buff, "The Statistical Mechanical Theory of Solutions. I," The Journal of Chemical Physics 19, 774 (1951).

${ }^{78}$ P. H. K. De Jong, J. E. Wilson, G. W. Neilson, and A. D. Buckingham, "Hydrophobic hydration of methane," Molecular physics 91, 99-104 (1997).

${ }^{79}$ F. Foglia, M. J. Lawrence, C. D. Lorenz, and S. E. McLain, "On the hydration of the phosphocholine headgroup in aqueous solution." The Journal of Chemical Physics 133, 145103 (2010).

${ }^{80}$ R. J. Gillams, J. V. Busto, S. Busch, F. M. Goñi, C. D. Lorenz, and S. E. McLain, "Solvation and hydration of the ceramide headgroup in a non-polar solution." The Journal of Physical Chemistry B 119, 128-139 (2015).

${ }^{81}$ J. J. Towey and L. Dougan, "Structural Examination of the Impact of Glycerol on Water Structure," The Journal of Physical Chemistry B 116, 1633-1641 (2012).

${ }^{82}$ A. K. Soper, "Probing the structure of water around biological molecules: concepts, constructs and consequences," Physica B: Condensed Matter 276-278, 12-16 (2000)

${ }^{83}$ J. L. Finney, D. T. Bowron, R. M. Daniel, P. A. Timmins, and M. A. Roberts, "Molecular and mesoscale structures in hydrophobically driven aqueous solutions," Biophysical Chemistry 105, 391-409 (2003).

${ }^{84}$ A. P. Dabkowska, F. Foglia, M. J. Lawrence, C. D. Lorenz, and S. E. McLain, "On the solvation structure of dimethylsulfoxide/water around the phosphatidylcholine head group in solution." The Journal of Chemical Physics 135, 225105 (2011). 